EDUCATIONAL

RESOURCE

Volume 12 Issue 32020

DOI: 10.21315/eimj2020.12.3.8

ARTICLE INFO

Submitted: 16-04-2020

Accepted: 20-05-2020

Online: 30-09-2020

\section{Five Tips for Managing Diagrams in Anatomy Lecture: The Cognitive Load Theory Perspective}

\author{
Siti Nurma Hanim Hadie
}

Department of Anatomy, School of Medical Sciences, Universiti Sains Malaysia, Kelantan, MALAYSIA

To cite this article: Hadie SNH. Five tips for managing diagrams in anatomy lecture: the cognitive load theory perspective. Education in Medicine Journal. 2020;12(3):65-74. https://doi.org/10.21315/ eimj2020.12.3.8

To link to this article: https://doi.org/10.21315/eimj2020.12.3.8

\title{
ABSTRACT
}

Managing diagrams for instructional materials especially for lecture is a thought-provoking task for educators. Poorly managed diagrams in lecture results in inefficient delivery of lecture content, while well-prepared diagrams would capture students' attention and facilitate their understanding. Anatomy is often perceived as a cognitively challenging subject for medical students but having adequate anatomy knowledge is indispensable for safe clinical practice. Since lecture is the most common method for anatomy teaching in many medical institutions, it is important to design a good anatomy diagram that can stimulate three-dimensional visualisation from two-dimensional instruction. Techniques of designing and preparing good diagrams can be adopted from an instructional design theory that is the cognitive load theory, which describes the occurrence of learning when designed instruction is aligned with human cognitive architecture and function. This paper provides a deeper insight on effective diagram management and outlines five tips on such endeavour based on the theory.

Keywords: Anatomy diagram, Lecture, Cognitive load theory Health Campus, Universiti Sains Malaysia, 16150 Kubang Kerian, Kelantan, Malaysia | Email: snurma@usm.my

\section{INTRODUCTION}

Anatomy has long been regarded as a pillar for medical education, whereby adequate anatomy knowledge is required for safe clinical practice (1). Ever since the major medical curricula revamp in the early 20th century, teaching and learning in anatomy underwent evolution to adapt with the changes (2). With reduction of anatomy content and teaching hours, cadaveric dissection which allows visuospatial manipulation of anatomical structures is no longer feasible in many medical schools (3), and thus anatomy teaching has to rely on other forms of visual aids to cater for visuospatial learning. There are many tools used for visuospatial learning in anatomy such as atlas illustration, online diagrams, living subjects, anatomy models, prosected specimens, radiographic images and augmented reality images (4-6). Among these visual aids, illustration and diagrams are the most commonly used by anatomists and medical students for teaching and learning anatomical structures respectively, as they are easily accessible.

Anatomy diagrams are often used in lecturebased instruction to facilitate information 
delivery of anatomy structures and their relations (7-8). It is arguable that lecture is still the most feasible face-to-face method to deliver information to a large group of students as it is cost-effective and not time consuming (9). In view of high criticism on lecturing method for not being effective, anatomy educators have introduced innovations into their lecturing methods to ensure efficient delivery of information and achievement of learning outcomes. These include incorporating evidence-based effective lecturing strategies (7-8), implementing flipped classroom by combining lectures with e-learning activities (9), integrating thought provoking facts at the beginning of lectures (10) and conducting intra-lecture activities to make the lecture interesting (11). Nevertheless, these efforts will not be fruitful if anatomy diagrams presented in the lecture are complicated and unintelligible. The central tenet of learning anatomy is to understand the anatomical structures, which can be achieved only when the learners are able to mentally visualise images of the anatomical structures. Hence, diagrams in lectures should be designed in a manner that can facilitate visuospatial learning.

To design an effective diagram for lecturebased instruction, it is imperative for anatomy educators to understand the basic structure of human cognitive function. As described by the Atkinson-Shiffrin's threestage information processing model of human cognition, there are three types of human memory, which include a sensory memory that receives new information from the external environment, a working memory that processes the information received form sensory memory, and a longterm memory that stores the information permanently (12). The process of information processing in human mind begins when the sensory memory receives new information from external environment, which can be in the form of visual, auditory, olfactory, taste or tactile stimulus (13). Despite its unlimited storage capacity, the sensory memory can hold a vast number of stimuli at one time, but within an ultrashort duration, which is less than one second for iconic memory (i.e., sensory memory for visual information) (14). Therefore, the stimulus must be quickly transferred to the working memory as to prevent it from decaying (14). Having said that, the information transfer process from the sensory to working memories requires presence of attention focus of the learner. When a person is consciously paying attention to one visual information out of many visual stimuli in his or her sensory memory, this visual information will be successfully transferred into the working memory (15).

In the working memory, this information is processed into an organised form of information, known as schema. Unlike sensory memory, the processing and storage capacity of working memory are limited in the sense that it can only receive seven plus minus two information at one time with an average retention time of 20 seconds (16). Hence, the schema decays if it is not transferred to the long-term memory for storage within the limited duration. On the other hand, if the schema is successful transferred to the long-term memory, it will be stored permanently, and the learner will experience the feeling of being able to understand the information. Hence, successful storage of schema by the long-term memory marks the occurrence of actual learning (17). These stored schemas can be retrieved back into the working memory to be incorporated with the newly received information from the sensory memory for subsequent schema construction (18). These forms of retrieved stored schema indicate prior knowledge.

With considerable evidence on the capacity of human cognitive architecture, many efforts were invested by educational psychology researchers in an attempt to find an efficient way of learning (19). This had led to the introduction of the cognitive load theory (CLT), an instructional design theory that describes the occurrence of actual learning when instructional materials 
are designed in manner that follows how the human mind works (20). The CLT outlines two types of cognitive loads, which are intrinsic and extraneous loads, and one cognitive resource, which is known as germane load. Intrinsic load refers to the complexity of an instruction, whereby it is imposed by number of elements present in the instruction and the interrelations that exist among them (21). For instance, a cranial nerve topic that is often perceived difficult by medical students imposes higher intrinsic load compared to a simple topic like anatomy of the tongue. This is because, there is more information under the cranial nerve topic and this information is linked to each other. Hence, a difficult topic is said to impose higher intrinsic load to the learner compared to a less difficult topic. Likewise, intrinsic load also depends on prior knowledge of a learner (22). A learner with prior knowledge on the presently learned subject experiences less intrinsic load than those who have no prior knowledge.

On the other hand, extraneous load is the unwanted load that can hinder learning (21). This load is imposed by poor organisation and delivery of instructional materials, as well as distraction from the external environment (21). An organised and well-delivered instruction imposes lower extraneous load to the learners compared to poorly designed instruction. Likewise, distraction imposes high extraneous load to learners because the available cognitive resources would be utilised to process the distraction. To ensure optimal learning, total cognitive load (i.e., summation of intrinsic and extraneous loads) must not exceed the limited capacity of the human working memory (21). If it is within the capacity of the human working memory, there will be adequate remaining cognitive resources (i.e., germane load) to process the intrinsic load, and thus resulted in the occurrence of learning.

The CLT has outlined several empirically proven strategies that can help educators in managing their students' intrinsic and extraneous loads (23-25). The intrinsic load can be managed by using a principle, known as the isolated interacting element effect. This principle suggests educators to chunk information into smaller components that are taught in isolation, followed with teaching the components together by introducing their relationship (24). Besides that, intrinsic load can be reduced if the learners have some prior knowledge before the formal class. In lecture environment, this condition could be materialised by conducting a pre-lecture activity that stimulates or instills prior knowledge of the learners (26). Likewise, the CLT also outlines several principles to reduce extraneous load, which include the modality effect, contiguity effect, split attention effect, redundancy effect, worked example effect and expertise reversal effect (23-25, 27-30). Some of these principles are elaborated in the following subheadings tips.

\section{TIPS OF DIAGRAM MANAGEMENT}

This article aims to provide worked examples of effective diagram management for lecture-based instruction, either through face-to-face mode or online platform. The strategies of diagram management that are proposed in this article incorporate the principles of the CLT, for managing the intrinsic load and reducing the extraneous load. If the strategies are successfully implemented, the total cognitive load of learners during lecture could be reduced, and therefore promotes learning. There are five tips for preparing and presenting diagram in lecture, which are: (a) select appropriate diagram, (b) include important labels only, (c) integrate related text, (d) introduce visual cues, and (e) explain diagram verbally.

\section{Tip 1: Select Appropriate Diagram}

Selecting diagrams, particularly for anatomy lecture should be done with caution as to ensure the diagram would reflect the actual topographic of the learned structure. Three important elements of diagram that need to 
be considered before selecting the diagram are size of the diagram, its colour and labels that are present in the diagram.

A diagram for lecture-based instruction must be appropriately sized. It should be sized appropriately so that it will clearly reveal the anatomical structures that are to be explained. In addition, space in the presentation tools (e.g., PowerPoint, Prezi, Visme and Scribe) must be reserved for related text to appear next to anatomical features when necessary. To ensure selection of an appropriately sized diagram, educators need to have digital skills in managing the diagrams. Most of the time, the diagrams are retrieved from the Internet, hence, skills in managing online diagrams such as converting diagrams to JPEG or PNG format is important to ensure good resolution of diagram when it is displayed. Magnifying a small-sized scanned or online diagram can result in a blurred image, and thus increase the extraneous load of learners.

In addition, coloured anatomy diagram is preferred compared to a black and white diagram. Coloured anatomy diagrams facilitate students' understanding of topographical relationship of structures, whereby the learners would be able to differentiate the learned structures from other related structures, through colours.
Moreover, coloured anatomy diagrams are vibrant and provide significant input to attract students' attention during the lecture. Nevertheless, it should be noted that the colours of the diagrams must be simple, clear and lack of visual shades. The colours should not be too bright as they can cause vibrating illusion, a form of distraction that imposes high extraneous load to the learners.

Apart from that, it is crucial to use an unlabelled diagram during lecture preparation for several reason as follows: (a) an unlabelled diagram appears clearer and gives more space in the presentation slide for other inputs, (b) lecturer is free to determine which labels need to be shown in the diagram by adding the labels manually (i.e., techniques for adding labels is described in the next subheadings), (c) a heavily labelled diagram imposes high extraneous load because the extraneous labels which are not required for understanding of the instructional contents become distractions to the learners. When a diagram with labels is retrieved from any source, it is important to remove the labels by using any digital diagram editing application, such as Microsoft Paint application. Figure 1 illustrates an appropriately sized coloured diagrams without label.

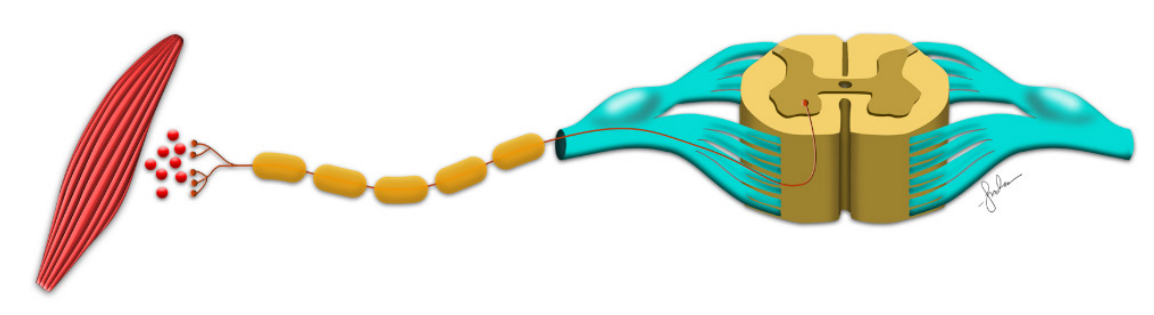

Figure 1: Example of a PowerPoint slide illustrating a coloured diagram without labels. The size of the diagram is appropriate as there is adequate space to insert text of labels. It is important to note that coloured diagram is suitable with white background, hence white background slide is suggested. 


\section{Tip 2: Include Important Labels Only}

As mentioned earlier, an anatomical diagram with many labels needs to be edited before it will be appropriate for use in a lecture. It is important to label only the structures under discussion, as doing so can reduce learners' extraneous loads. Hence, the labels should be aligned with the verbal explanation or related text narration of the diagram. Labels of structures that are not mentioned in the verbal explanation or related text narration should be removed, even though they are the labels of important structures. Figure 2 illustrates the diagram that has been labelled accordingly based on the related textual narration.

\section{Somatic motor innervation}

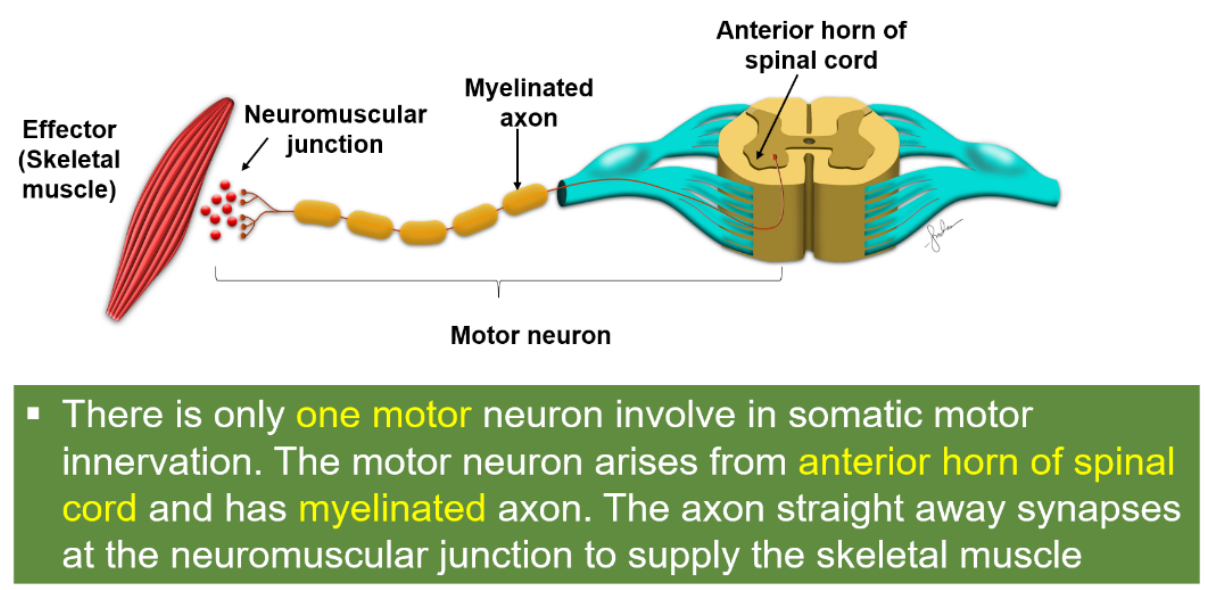

Figure 2: Example of a PowerPoint slide illustrating a diagram that is labelled sufficiently to accommodate the text narration. All the structures labelled are mentioned in the text.

\section{Tip 3: Integrate Related Text}

Self-explanatory diagrams should be avoided when presenting material to novice learners, who have been demonstrated to be the least intelligible format for inexperienced learners when compared to diagrams with textual narration, diagrams with verbal explanation, and diagrams with both textual narration and verbal explanation. If the learners have already gained some experience on the subject matter, however, self-explanatory diagrams can be used, since they have been shown to be effective under such circumstances. This condition is known as the expertise reversal effect of the CLT (30).

Since anatomy is a subject that is often taught to the pre-clinical year students, who are novices in anatomy knowledge, presentation of diagrams should be accompanied with textual narration and verbal explanation. Textual narration should be concise and follow the "seven plus minus two" rules of human working memory (31). Nevertheless, it is arguable that the human working memory can only process three to five chunks of information at one time (16). Hence, it is advisable that the number of information contained in the text narration should be limited within the range between three to five, considering diagram is also a visual information that needs to be captured by human memory. In addition, the textual narration should not be wordy. It should be written using San-Serif font (i.e., Arial, Calibri, Tahoma and Halvetica), which appears clearer when displayed on digital screens compared to Serif font (i.e., Times New Roman, Bookman Old Style, Cambria 
and Georgia). The font size of the textual narration should not be less than 22-point size.

Moreover, it is important to note that textual narration should be placed in the same presentation slide as suggested by the spatial contiguity effect of the CLT. This principle describes optimisation of learning when two related visual information (i.e., diagrams and textual narration) is placed near to each other (29). This technique aims to prevent the split attention effect that increases the extraneous load of learners, as they have to split their attention into two related information if these information are spatially presented (28). Figure 2 illustrates an example on how to write a textual material.

\section{Tip 4: Introduce Visual Cues}

Incorporating visual cues and signals into the diagram can help learners to capture and focus their attention on important pictorial information. For example, a lecturer can use arrows, circles or animation to draw learners' attention towards a certain part of the diagram. Labels of the structures can be made animated (i.e., labels appear with mouse click) to synchronise the learners' attention with verbal explanation. Moreover, when designed and planned properly, these cues and signals can enhance learners' understanding of a complex and lengthy instructions, but precaution should be taken to avoid element overcrowding in any given slide. For instance, if a slide includes too many cues or animation, it will distract rather than guide the learners, thus hampering learning. The technique of using visual cues fulfils a principle of cognitive theory of multimedia learning (CTML), known as signalling principle (32). It describes that with the use of visual cues, the learners' attention will be directed towards essential material for schema acquisition and construction $(33,34)$. This indirectly reduces extraneous cognitive processing of the learners (32). Figures 3 and 4 illustrate the use of visual cues in anatomy diagrams.

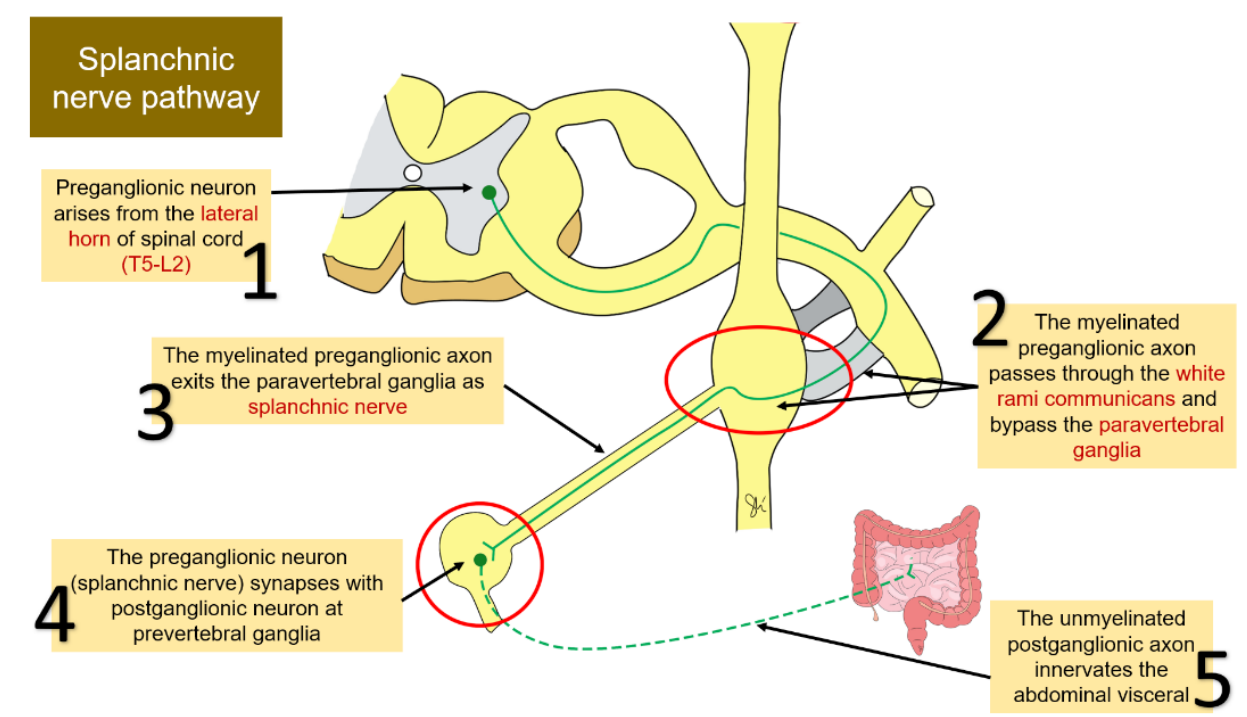

Figure 3: Visual cues in the forms of red circles, arrows and numbers are used to guide learners on the sequence of information presentation. 


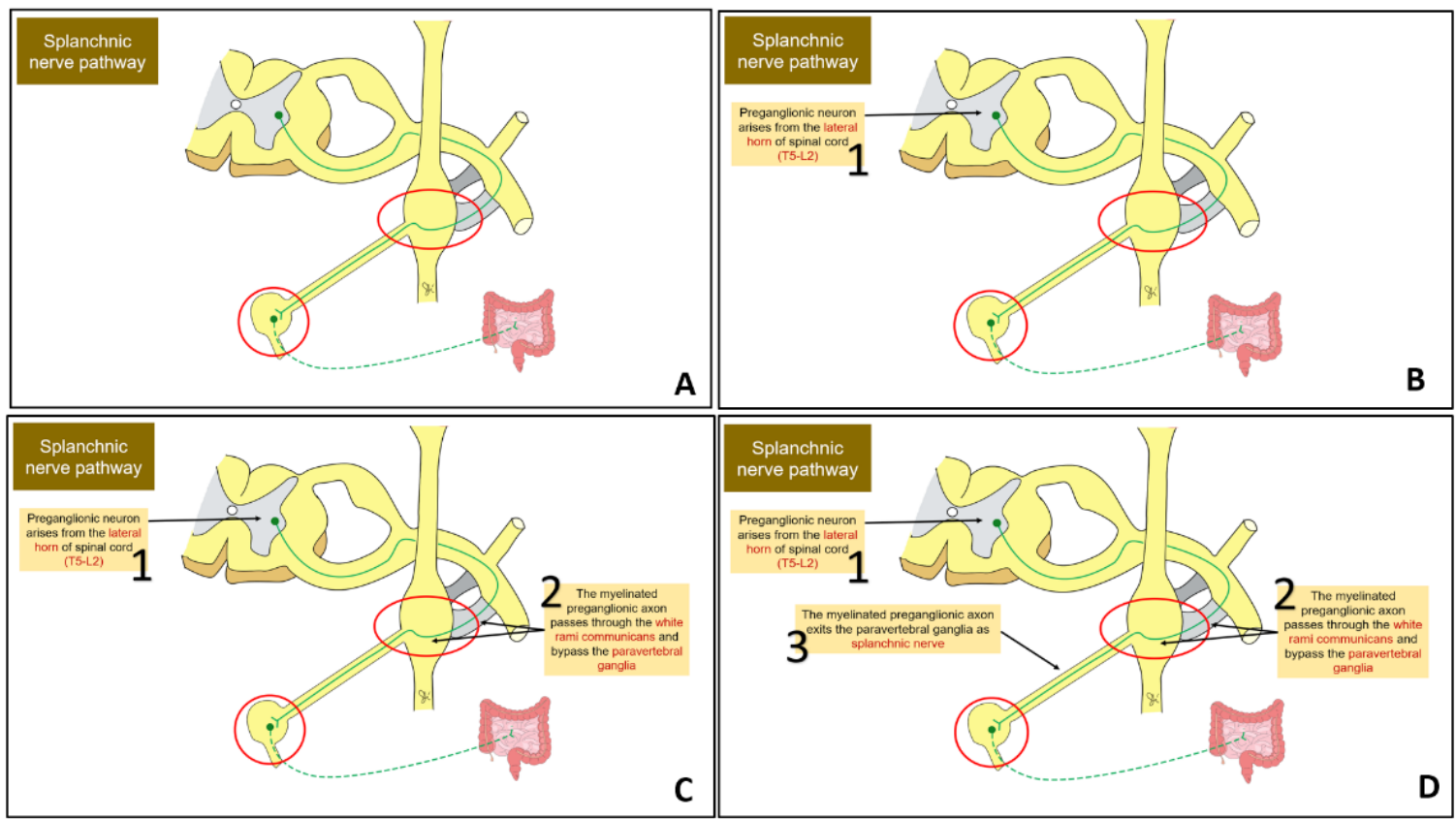

Figure 4: These four different presentation slides illustrate the use of animated labels in anatomy diagrams. At the beginning of the diagram presentation, there is no label shown (Slide A). Each label was made visible one at a time (Slides B, C and D), aligning with lecturer's verbal explanation.

\section{Tip 5: Explain Diagram Verbally}

The final step of diagram management is to present the diagram verbally by using own words. As mentioned earlier, anatomy diagram of lecture-based instruction should not be self-explanatory, as the pre-clinical year medical students are mostly novices. Although textual materials are presented with the diagram in a same presentation slide, it is important to highlight that reading from the text is not advisable. According to the CLT, concurrent presentation of both words and pictures that are presented verbally would simultaneously stimulate the visual and auditory centres of the learner's working memory (35). Hence, the working memory resources are being optimised. This condition is known as the temporal contiguity effect of the CLT. Reading verbatim from the slides would increase learners' cognitive load as the students will only use their visual centre, as the lecturer's voice is being perceived as a noise, and thus eventually would result in visual centre overload. This condition is known as redundancy effect and would increase learners extraneous load during lecture (27).

\section{CONCLUSION}

Managing diagram for lecture-based instruction is important especially for difficult subject like anatomy that requires 3-dimensional visualisation and visuaspatial learning. Application of the CLT in diagram management would provide extra credential to the lecturing process, due to various factors. First, CLT is based on human cognition framework, which from that basis, it outlines emprically-proven teaching strategies for effective learning to occur. Second, the CLT principles prevent information overload, which is a common situation during lecture. Third, the CLT principles minimise wasted mental resources as they cater more for reduction of extraneous rather than intrinsic loads. The wasted mental resources are not only minimised but are put to work for the purposes of information processing, and therefore lead to faster and better learning. 
Fourth, the CLT principles are universal and practical. Although this paper focusses on diagram management for lecture-based instruction in anatomy, the same principles can be applied to other form of instruction such as in e-learning or massive open online course - and disciplines. The five tips of diagram management outlined in this paper are doable and practical. If properly applied, the diagram presentation during lecture will be interactive and enhance the students' understanding of the learned topic.

\section{ACKNOWLEDGEMENTS}

The author would like to thank $\mathrm{Mr}$ Muhamad Nor Firdaus Ab Rahman and Madam Norhana Arshad, medical laboratory technologist from Department of Anatomy, Universiti Sains Malalysia for their contributions in drawing two anatomy diagrams that are reported in this article.

\section{REFERENCES}

1. Singh R, Tubbs RS, Gupta K, Singh $M$, Jones DG, Kumar R. Is the decline of human anatomy hazardous to medical education/profession?-A review. Surg Radiol Anat. 2015;37(10):1257-65. https:// doi.org/10.1007/s00276-015-1507-7

2. Davies D, Malak S, Jensen J, Akhter N, Beneš $\mathrm{H}$, Childs $\mathrm{G}$, et al. Medical gross anatomy: conversion of a traditional discipline-based course to a briefer and more clinically focused foundational module in a revamped preclinical curriculum (532.1). FASEB J. 2014;28(S1):531-2.

3. Bay $\mathrm{BH}$, Ling EA. Teaching of anatomy in the new millennium. Singapore Med J. 2007;48(3): 182 .

4. Malone ER, Pine M, Bingham G. A kinetic model for learning gross anatomy. FASEB J. 2016;30(S1):782-4.
5. Estai M, Bunt S. Best teaching practices in anatomy education: a critical review. Ann Anat. 2016;208:151-7. https://oi. org/10.1016/j.aanat.2016.02.010

6. Karambakhsh A, Kamel A, Sheng B, Li P, Yang P, Feng DD. Deep gesture interaction for augmented anatomy learning. Int J Inf Manage. 2019;45:328-36. https://doi. org/10.1016/j.ijinfomgt.2018.03.004

7. Hadie SNH, Abdul Manan@Sulong H, Hassan A, Mohd Ismail ZI, Talip S, Abdul Rahim AF. Creating an engaging and stimulating anatomy lecture environment using the cognitive load theory-based lecture model: students' experiences. J Taibah Univ Med Sci. 2018;13(2):162-72. https://doi. org/10.1016/j.jtumed.2017.11.001

8. Hadie SNH, Hassan A, Mohd Ismail ZI, Ismail HN, Talip SB, Abdul Rahim AF. Empowering students' minds through a cognitive load theory-based lecture model: a metacognitive approach. Innov Educ Teach Int. 2018;55(4):398-407. https://doi.org/10 .1080/14703297.2016.1252685

9. Lochner L, Wieser H, Waldboth S, MischoKelling M. Combining traditional anatomy lectures with e-learning activities: how do students perceive their learning experience? Int J Med Educ. 2016;7:69-74. https://doi. org/10.5116/ijme.56b5.0369

10. Narayanan S, Shankar N, Ananthy V. Medical student's perception to different types of set induction in anatomy lectures. Anat Cell Biol. 2019;52(3):296-301. https:// doi.org/10.5115/acb.19.016

11. Kotzé SH, Mole CG. Making large class basic histology lectures more interactive: the use of draw-along mapping techniques and associated educational activities. Anat Sci Educ. 2015;8(5):463-70. https://doi. org/10.1002/ase.1514

12. Klahr D, MacWhinney B. Information processing. Handb Child Psychol. 1998;2:631-78. 
13. Carlson NR, Miller H, Heth CD, Donahoe JW, Martin GN. Psychology: the science of behavior. 7th ed. Boston, MA: Allyn \& Bacon (Pearson); 2010.

14. Bradley C, Pearson J. The sensory components of high-capacity iconic memory and visual working memory. Front Psychol. 2012;3:355. https://doi.org/10.3389/ fpsyg.2012.00355

15. DiMaggio P. Culture and cognition. Annu Rev Sociol. 1997;23(1):263-87. https://doi. org/10.1146/annurev.soc.23.1.263

16. Cowan $\mathrm{N}$. The magical mystery four: how is working memory capacity limited, and why? Curr Dir Psychol Sci. 2010;19(1):51-7. https://doi.org/10.1177/0963721409359277

17. Ericsson KA, Kintsch W. Longterm working memory. Psychol Rev. 1995;102(2):211. https://doi. org/10.1037/0033-295X.102.2.211

18. Brod G, Lindenberger U, Shing YL. Neural activation patterns during retrieval of schema-related memories: differences and commonalities between children and adults. Dev Sci. 2017;20(6):e12475. https://doi. org/10.1111/desc. 12475

19. Sweller J. Cognitive load during problem solving: effects on learning. Cognitive Science. 1988;12(2):257-85. https:/doi. org/10.1207/s15516709 $\operatorname{cog} 1202 \_4$

20. Chandler P, Sweller J. Cognitive load theory and the format of instruction. Cognition and Instruction. 1991;8(4):293-332. https://doi. org/10.1207/s1532690xci0804_2

21. Sweller J. Element interactivity and intrinsic, extraneous, and germane cognitive load. Educ Psychol Rev. 2010;22(2):123-38. https://doi.org/10.1007/s10648-010-9128-5

22. Cook MP. Visual representations in science education: the influence of prior knowledge and cognitive load theory on instructional design principles. Sci Educ. 2006;90(6):1073-91. https://doi. org/10.1002/sce. 20164
23. Ginns P. Meta-analysis of the modality effect. Learning Instruction. 2005;15(4):313-31. https://doi. org/10.1016/j.learninstruc.2005.07.001

24. Blayney P, Kalyuga S, Sweller J. Interactions between the isolated-interactive elements effect and levels of learner expertise: experimental evidence from an accountancy class. Instructional Science. 2010;38(3):277-87. https://doi.org/10.1007/ s1 1251-009-9105-x

25. Paas F, Van Gog T. Optimising worked example instruction: different ways to increase germane cognitive load. Learning and Instruction. 2006;16(2):87-91. https:// doi.org/10.1016/j.learninstruc.2006.02.004

26. Hadie SNH, Simok AA, Shamsuddin SA, Mohammad JA. Determining the impact of pre-lecture educational video on comprehension of a difficult gross anatomy lecture. J Taibah Univ Med Sci. 2019;14(4):395-401. https://doi. org/10.1016/j.jtumed.2019.06.008

27. SchüLer A, Scheiter K, Gerjets P. Is spoken text always better? Investigating the modality and redundancy effect with longer text presentation. Comput Human Behav. 2013;29(4):1590-601. https://doi. org/10.1016/j.chb.2013.01.047

28. Cierniak G, Scheiter K, Gerjets P. Explaining the split-attention effect: is the reduction of extraneous cognitive load accompanied by an increase in germane cognitive load? Comput Human Behav. 2009;25(2):315-24. https://doi. org/10.1016/j.chb.2008.12.020

29. Ginns P. Integrating information: a metaanalysis of the spatial contiguity and temporal contiguity effects. Learning Instruction. 2006;16(6):511-25. https://doi. org/10.1016/j.learninstruc.2006.10.001 
30. Kalyuga S, editor. The expertise reversal effect. In: Managing cognitive load in adaptive multimedia learning. Pennsylvania, U.S.: IGI Global; 2009. p. 58-80. https:// doi.org/10.4018/978-1-60566-048-6.ch003

31. Linden DEJ. The working memory networks of the human brain. Neurosci. 2007;13(3):257-67. https://doi.org/10.11 $77 / 1073858406298480$

32. Sorden SD. The cognitive theory of multimedia learning. Handb Educ Theor. 2012;1:31.

33. Lin L, Atkinson RK. Using animations and visual cueing to support learning of scientific concepts and processes. Comput Educ. 2011;56(3):650-8. https://doi.org/10.1016/j. compedu.2010.10.007
34. Xie H, Wang F, Hao Y, Chen J, An J, Wang $\mathrm{Y}$, et al. The more total cognitive load is reduced by cues, the better retention and transfer of multimedia learning: a metaanalysis and two meta-regression analyses. PLoS One. 2017;12(8):e0183884. https:// doi.org/10.1371/journal.pone.0183884

35. Sederberg PB, Miller JF, Howard MW, Kahana MJ. The temporal contiguity effect predicts episodic memory performance. Mem Cognit. 2010;38(6):689-99. https:// doi.org/10.3758/MC.38.6.689 